\title{
Asymptotic Solutions of the Restricted Three-Body Problem by Use of Perturbation Methods
}

\author{
By Zeal-Sain KuO \\ Department of Mechanical Engineering, Chung Cheng Institute of Technology, National Defense University, Taiwan, R.O.C.
}

(Received November 5th, 2001)

\begin{abstract}
This work presents a comparison of matching asymptotic solutions for the limiting case of the restricted threebody problem by the use of perturbation methods. The problem is of a singular-perturbation type. We investigate two alternative methods to deal with it: the classical method of matched asymptotic expansions and the improved method of matched asymptotic expansions. Two expansions, outer and inner, are involved. The outer expansion breaks down in the inner region where sharp changes occur, and the inner expansion becomes nonuniformly valid in the outer region. To obtain a uniformly valid composite solution, we need a matching procedure to relate these two expansions. Instead of straightforward matching of the outer and inner expansions to higher-order terms, in the improved technique the higher-order solutions are derived by generating perturbations between the lower-order composite solutions and the exact solutions. The perturbation equations are then integrated in the outer and inner regions, respectively, for a higher-order matching. Improved asymptotic solutions of second order are obtained for the limiting case of the restricted three-body problem. Compared to the solutions obtained by the classical method of matched asymptotic expansions and the pure numerical integration for various values of a small parameter $\mu$, the improved asymptotic solutions are very accurate. Moreover, the asymptotic solutions obtained by use of the improved method give better accuracy than those using the classical method over wide ranges of the small parameter.
\end{abstract}

Key Words: Restricted Three-Body Problem, Perturbation Methods, Matched Asymptotic Expansions, Singular Perturbation

\section{Introduction}

The planar, circular, restricted three-body problem is often used to study the motion of interplanetary trajectories, since it describes most orbital problems of nature for astronautical phenomena and applications. ${ }^{1)}$ The research of the restricted three-body problem thus received sizable interest through the use of numerical and analytical approaches. ${ }^{2-5)}$ Our present knowledge of the motions of the solar system and our great physical database of it, together with the huge capacities of modern computers, allows us to obtain extremely accurate trajectories for space missions. However, the major defect of these numerical methods is that numerical solutions are usually confined to certain parameters and conditions; that is, no general property, even an approximate one, can be made from the results of the numerical computations of several trajectories. The analytical methods are much less accurate, but they give a general picture of the set of orbits and thus allow us to choose a set of parameters and conditions for numerical computation according to the mission. In this paper, a comparison of matching asymptotic solutions for the limiting case of the restricted three-body problem by the use of perturbation methods is presented. The problem is of a singular-perturbation type. We investigate two alternative methods to deal with it: the classical method of matched asymptotic expansions (MAE) and the improved method of MAE.

(c) 2002 The Japan Society for Aeronautical and Space Sciences
The method of MAE (inner and outer expansions) was initially developed by Prandtl (1905) for solving the problem of high speed viscous flow past a body. ${ }^{6}$ Extended and generalized over a long time, this method has been formalized by many researchers including Van Dyke, ${ }^{7)}$ Kaplun and Lagerstrom, ${ }^{8)}$ and Kevorkian and Cole. ${ }^{9)}$ In his book, Nayfeh presented the basic ideas of the matching procedures for the method. ${ }^{10)}$ Using specific examples, Lagerstrom brought out its essential concepts and techniques. ${ }^{11)}$ After Lagerstrom and Kevorkian ${ }^{12)}$ applied it to study earth-to-moon trajectories, Breakwell and Perko ${ }^{13)}$ and other astrodynamicists studied the applications of this technique in interplanetary trajectories. The implementation of the method of MAE has opened new opportunities of research in celestial mechanics and other fields. Two expansions are involved in the method, namely, the outer expansion and the inner expansion. The outer expansion breaks down in the inner region where sharp changes occur, and the inner expansion becomes nonuniformly valid in the outer region. To obtain a uniformly valid composite solution, a matching procedure is used to relate these two expansions. The asymptotic solutions derived by this method, called composite solutions, are formulated by totaling the outer and the inner expansions and subtracting the common limits under an appropriate matching principle. In general, the classical method of MAE is applied to solve two-boundary value problems (2BVP). When we apply the MAE method to initial value problems (IVP), because of the propagation of errors along the trajectory and the crossover 
interference between different order terms in the matching process, the resulting accuracy usually depends on the property of the physical problems.

On the other hand, in the improved MAE method (iMAE), ${ }^{14,15)}$ instead of a straightforward matching of the outer and inner expansions to higher-order terms, the higherorder solutions are derived first by generating perturbations between the lower-order composite solutions and the exact solutions. The perturbation equations are then integrated in the outer and inner regions, and a higher-order matching is performed for the expansions of the perturbations. The higher-order solutions are thus the sum of the lower-order solutions and the composite solutions of the perturbations.

The method is powerful because of its inherent property that incorporates the knowledge of physical characteristics at the two-end boundaries of the interplanetary trajectory and rigorous mathematical procedures. Improved asymptotic solutions of a second order are obtained for the limiting case of the restricted three-body problem and compared to the solutions obtained by the classical method of MAE and the pure numerical integration over wide ranges of the mass ratio.

\section{Equations of Motion}

Consider the planar, circular, restricted three-body problem as shown in Fig. 1. The mass of the spacecraft is much smaller than the other two bodies, and the mass $M_{1}$ (for convenience referred to as the earth) is much larger than the mass $M_{2}$ (the moon). In the gravitational field of two-fixed mass centers, the equation of motion for the spacecraft is given as ${ }^{12)}$

$$
\frac{\mathrm{d}^{2} \tilde{\boldsymbol{r}}}{\mathrm{d} \tilde{t}^{2}}=\operatorname{Ggrad}\left[\frac{M_{1}}{\tilde{r}_{1}}+\frac{M_{2}}{\tilde{r}_{2}}\right]
$$

where

$$
\tilde{r}_{1}^{2}=\tilde{x}^{2}+\tilde{y}^{2}, \text { and } \tilde{r}_{2}^{2}=(\tilde{x}-d)^{2}+\tilde{y}^{2}
$$

$G$ is the universal gravitational constant, and $d$ is the distance between the two mass centers. We define the dimensionless parameter as the mass ratio.

$$
\mu=\frac{M_{2}}{M_{1}+M_{2}}
$$

The position vector $(\tilde{x}, \tilde{y})$ was normalized by the distance $d$ between the two mass centers. The time was made dimensionless by use of the distance $d$ and the factor

$$
\frac{d^{3 / 2}}{\sqrt{G\left(M_{1}+M_{2}\right)}}
$$

The equations of motion with respect to the rectangular Cartesian coordinate system in dimensionless form become

$$
\begin{aligned}
& \frac{\mathrm{d}^{2} x}{\mathrm{~d} t^{2}}=-(1-\mu) \frac{x}{r_{1}{ }^{3}}+\mu \frac{1-x}{r_{2}{ }^{3}} \\
& \frac{\mathrm{d}^{2} y}{\mathrm{~d} t^{2}}=-(1-\mu) \frac{y}{r_{1}{ }^{3}}-\mu \frac{y}{r_{2}{ }^{3}}
\end{aligned}
$$

This problem has been studied by Lagerstrom and Kevorkian ${ }^{12)}$ and $\mathrm{Nayfeh}^{10)}$ for the initial conditions

$$
x=0, y=0, \frac{\mathrm{d} y}{\mathrm{~d} x}=-\mu c \text { and } q=-\rho^{2} \text { at } t=0
$$

where $c$ is a constant with order $O(1), q$ is the total energy of the spacecraft, and $\rho \neq 1$. It is expected that the integrals of the Keplerian motion near the earth will play an important role for solving this problem, since the Keplerian integrals of the energy and angular momentum will change very little near the earth. The integrals are listed as follows:

$$
\begin{aligned}
& q_{\mathrm{E}}=\frac{1}{2}\left[\left(\frac{\mathrm{d} x}{\mathrm{~d} t}\right)^{2}+\left(\frac{\mathrm{d} y}{\mathrm{~d} t}\right)^{2}\right]-\frac{1-\mu}{r} \\
& h_{\mathrm{E}}=x \frac{\mathrm{d} y}{\mathrm{~d} t}-y \frac{\mathrm{d} x}{\mathrm{~d} t}
\end{aligned}
$$

where $q_{\mathrm{E}}$ and $h_{\mathrm{E}}$ are the energy and angular momentum relative to the earth. It is convenient to prescribe the initial conditions at $x=0$, and therefore the energy and angular momentum will stay constant for any $x=O(\mu)$. Thus we let

$$
q_{\mathrm{E}}=-\rho^{2}, \quad h_{\mathrm{E}}=h
$$

To make the initial conditions unique $y>0$ and to satisfy the integrals (7-8) and the conditions (9) exactly, we will have the initial values for $y$ and components of the velocity

$$
y=\frac{h^{2}}{1-\mu}, \quad \frac{\mathrm{d} x}{\mathrm{~d} t}=\frac{1-\mu}{|h|},
$$

and

$$
\frac{\mathrm{d} y}{\mathrm{~d} t}=\frac{1}{h} \sqrt{(1-\mu)^{2}-2 \rho^{2} h^{2}}
$$

for $x=0$ at $t=0$. As in Ref. 10, we shall use $x$ as the independent variable and transform the equations of motion into

$$
\begin{aligned}
& -\frac{t^{\prime \prime}}{t^{\prime 3}}+(1-\mu) \frac{x}{r_{1}{ }^{3}}=\mu \frac{1-x}{r_{2}{ }^{3}} \\
& \frac{y^{\prime \prime}}{t^{\prime 2}}-\frac{t^{\prime \prime} y^{\prime}}{t^{\prime 3}}+(1-\mu) \frac{y}{r_{1}{ }^{3}}=-\mu \frac{y}{r_{2}{ }^{3}}
\end{aligned}
$$

Equations (11) constitute the most appropriate form of equations for analyzing the motion of the restricted three-body problem by use of the perturbation methods.

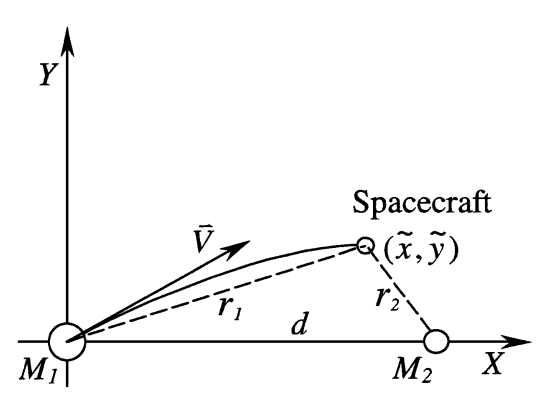

Fig. 1. Geometry of the restricted three-body problem. 


\section{Asymptotic Solutions near the Earth}

It has been shown that the straightforward perturbation expansion is not uniformly valid for all regions because of a logarithmic singularity at the position of the moon $\left(M_{2}\right)$. One technique to handle this problem is the method of matched asymptotic expansions, or called the method of inner and outer expansions. The outer expansion is to determine the straightforward expansion by the use of the original variables while the inner expansion determines the expansion where sharp changes occur using magnified scales. As long as $M_{2}$ is small, the right-hand sides of Eq. (11) represent small perturbations of order $\mu$. If these perturbations are neglected, Eq. (11) becomes the Kepler's equations of motion. We thus assume outer expansions of the form

$$
\begin{aligned}
& y=y_{0}(x, \mu)+\sum_{n=1}^{\infty} \mu^{n} y_{n} \\
& t=t_{0}(x, \mu)+\sum_{n=1}^{\infty} \mu^{n} t_{n}
\end{aligned}
$$

where the function $y_{0}(x, \mu)$ and $t_{0}(x, \mu)$ represent the exact solutions of the Keplerian motion derived by substituting the outer expansion for $y$ and $t$ in Eq. (11) and taking the outer limit as $\mu$ approaches zero. Thus the solutions of the first terms in the outer expansions satisfying the initial condition (10) exactly can be obtained. For the elliptic case $(\rho \neq 0)$, we have

$$
\begin{aligned}
y_{0}(x, \mu)= & -\frac{h}{(1-\mu)} \sqrt{h^{2}+2 e(1-\mu) x-2 \rho^{2} x^{2}} \\
t_{0}(x, \mu)= & \frac{(1-\mu)}{2 \sqrt{2} \rho^{3}} \sin ^{-1}\left[\sqrt{2} \rho \sqrt{\frac{2 e x}{(1-\mu)}+\frac{h^{2}-2 \rho^{2} x^{2}}{(1-\mu)^{2}}}\right] \\
& -\frac{e}{2 \rho^{2}} \sqrt{h^{2}+2 e(1-\mu) x-2 \rho^{2} x^{2}}
\end{aligned}
$$

where $e$ is the eccentricity of the elliptical orbit

$$
e=\sqrt{1-\frac{2 \rho^{2} h^{2}}{(1-\mu)^{2}}}
$$

Note that $h<0$ for positive $y$. Taking the direct expansion with respect to $\rho$ and letting the $\rho$ approaches to zero yield the parabolic case

$$
\begin{gathered}
y_{0}(x, \mu)=-h \sqrt{\frac{2 x}{(1-\mu)}+\frac{h^{2}}{(1-\mu)^{2}}} \\
t_{0}(x, \mu)=\frac{h^{2}}{2(1-\mu)} \sqrt{\frac{2 x}{(1-\mu)}+\frac{h^{2}}{(1-\mu)^{2}}} \\
-\frac{(1-\mu)}{6} \sqrt{\left(\frac{2 x}{(1-\mu)}+\frac{h^{2}}{(1-\mu)^{2}}\right)^{3}}
\end{gathered}
$$

To obtain uniformly valid asymptotic solutions for the inner region, we must now recognize and utilize the fact that the sharp changes are characterized by magnified scales that are different from the scale describing the behavior of dependent variables in the outer region. The proposed stretching transformation is

$$
\varphi=\frac{1-x}{\mu^{\alpha}}, \quad \psi=\frac{y}{\mu^{\alpha}}, \quad s=\frac{t-t_{\mathrm{e}}}{\mu^{\beta}}
$$

where $\alpha, \beta>0$, and $t_{\mathrm{e}}$ is the time elapsed to reach $x=1$. It can be shown by an analysis of the order of magnitude of the various terms in the equations that $\alpha=1$ and $\beta=$ 1. With the new variables, $\varphi=(l-x) / \mu$ and $\psi=y / \mu$, the original equations can be rewritten in terms of the new variables (inner variables) as

$$
\mu \frac{\mathrm{d} \varphi^{2}}{\mathrm{~d} t^{2}}=\frac{(1-\mu)(1-\mu \varphi)}{\left[(1-\mu \varphi)^{2}+(\mu \psi)^{2}\right]^{3 / 2}}+\frac{\mu^{2} \varphi}{\left[(\mu \varphi)^{2}+(\mu \psi)^{2}\right]^{3 / 2}}
$$

$$
\frac{\mathrm{d} \psi^{2}}{\mathrm{~d} t^{2}}=\frac{-(1-\mu) \psi}{\left[(1-\mu \varphi)^{2}+(\mu \psi)^{2}\right]^{3 / 2}}-\frac{\mu \psi}{\left[(\mu \varphi)^{2}+(\mu \psi)^{2}\right]^{3 / 2}}
$$

Theoretically, the asymptotic solutions for higher-order terms can be obtained step by step by the use of direct integration of the inner and outer expansions. Because in this paper we intend to compare the classical MAE method with the improved method, we focus on demonstrating the procedures of the improved technique. To facilitate the comparison, we now consider a one-dimensional case with the same initial conditions in Eq. (6). If we set $y=0$ and integrate Eq. (11) with the total energy $\rho$ being equal to zero (parabolic case), we have

$$
-\frac{t^{\prime \prime}}{t^{\prime 3}}+(1-\mu) \frac{1}{x^{2}}=\mu \frac{1}{(1-x)^{2}}
$$

and

$$
-\int \frac{\mathrm{d} t^{\prime}}{t^{\prime 3}}=\int\left[-(1-\mu) \frac{1}{x^{2}}+\mu \frac{1}{(1-x)^{2}}\right] \mathrm{d} x
$$

Integrating subject to the initial condition for the parabolic case, this yields

$$
\frac{1}{2 t^{\prime 2}}=\frac{(1-\mu)}{x}+\frac{\mu}{1-x}
$$

or we rewrite it in the form

$$
\frac{\mathrm{d} t}{\mathrm{~d} x}=\sqrt{\frac{x(1-x)}{2 \mu x+2(1-\mu)(1-x)}}
$$

Substituting the outer expansion for $t$ in Eq. (25) and using the outer limit, $\mu \rightarrow 0$, with $x$ fixed, the first term of the outer expansion is

$$
t_{0}=\frac{2}{3 \sqrt{2}} x^{3 / 2}+C_{0}
$$

where $C_{0}$ is the constant of integration. It should be noted that this solution can be easily obtained by taking the limiting case of $\mu \rightarrow 0$ and $h \rightarrow 0$ in the expansions of Eq. (18).

For the inner solution, the one near $x=1$, if we let $\psi=0$ in Eq. (20) and integrate the resulting equation, we obtain 


$$
\left(\mu \frac{\mathrm{d} \varphi}{\mathrm{d} t}\right)^{2}=\frac{2(1-\mu)}{1-\mu \varphi}+\frac{2}{\varphi}
$$

or rewrite it in the form

$$
\frac{\mathrm{d} t}{\mathrm{~d} \varphi}=\mu \sqrt{\frac{\varphi(1-\mu \varphi)}{2(1-2 \mu) \varphi+2}}
$$

This form is suitable for integration in the inner region. It is clear that the first term of the inner solution is the constant of integration because the $O(1)$ term on the right-hand side of Eq. (28) is zero. Therefore we have

$$
\tau_{0}=\mathrm{constant}
$$

where $\tau_{0}$ is the first term of the inner expansion

$$
t^{i}=\sum_{n=0}^{\infty} \mu^{n} \tau_{n}
$$

\section{First-Order Solutions}

To have the solutions uniformly valid over entire earthto-moon trajectory, we construct the composite solutions by totaling the first-order outer and inner solutions and subtracting the parts they have in common.

$$
s_{\mathrm{c}}=t_{0}+\tau_{0}-s_{00}
$$

subject to the initial condition $s_{\mathrm{c}}(0)=0$, where $s_{00}$ is the common limit of the inner and outer solutions. The basic matching principle for the first-order solutions is given as follows.

$$
s_{00}=\lim _{\substack{\mu \rightarrow 0 \\ x \text { fixed }}}\left(t_{0}\right)^{\mathrm{i}}=\lim _{\substack{\mu \rightarrow 0 \\ \varphi \text { fixed }}}\left(\tau_{0}\right)^{\mathrm{o}}
$$

which is equivalent to equating the inner limit of (the outer solution) denoted by $\left(t_{0}\right)^{\mathrm{i}}$ to the outer limit of (the inner solution) denoted by $\left(\tau_{0}\right)^{\mathrm{o}}$. Because the first term of the inner expansion is a constant, the first-order composite solution is identical to the outer solution because of a cancellation of the constant $C_{0}$ in the matching process. Thus we easily obtain the first-order composite solution.

$$
s_{\mathrm{c}}=\frac{2}{3 \sqrt{2}} x^{3 / 2}
$$

\section{Second-Order Composite Solutions}

Basically, we can proceed to derive the outer and inner expansions to a higher order in $\mu$, but as mentioned, because of error propagation and crossover interference between firstorder and second-order terms in the matching process, resulting errors may exceed the correction capability for the higher-order expansions when we use the classical method of MAE. In the improved technique, we obtain the secondorder composite solution by generating a perturbation between the first-order composite solution and the exact solution, and we then integrate the perturbation in the outer and inner regions again for a second-order matching. Let the perturbation $p$ be the difference between the exact solution $t$ and the first-order solution $s_{\mathrm{c}}$

$$
p=t-s_{\mathrm{c}}
$$

By substituting of Eq. (34) in Eq. (25), we obtain the equations for the small perturbation $p$

$$
\begin{aligned}
\frac{\mathrm{d} p}{\mathrm{~d} x} & =\sqrt{\frac{x(1-x)}{\mu x+(1-\mu)(1-x)}}-\sqrt{x} \\
& =\mu \sqrt{x}-\frac{\mu \sqrt{x}}{2(1-x)}+\frac{3 \mu^{2}}{8} \sqrt{x}\left(\frac{1}{1-x}-2\right)^{2}+\text { H.O.T. }
\end{aligned}
$$

subject to the initial condition

$$
p(0)=0
$$

\subsection{Outer expansion (Regular region)}

In the neighborhoods of the earth $\left(M_{1}\right)$, the motion of the spacecraft is dominated by earth gravity because the gravitational force of the moon is negligible compared to earth gravity. The outer expansion is just the straightforward expansion that uses the original variables. Therefore the outer expansion is obtained by repeated application of the outer limit, which is defined as the limit when $\mu \rightarrow 0$ with the variable $x$ and other dimensionless quantities held fixed. Because the right-hand side of Eq. (35) is of $O(\mu)$, we expect an outer expansion in the form.

$$
p^{\mathrm{o}}=\sum_{n=1}^{\infty} \mu^{n} p_{n}
$$

Substituting the outer expansion for $p$ in Eq. (35) and integrating the $O(\mu)$ terms of the outer expansion, it can be easily shown that the outer expansion is

$$
\begin{aligned}
p^{\mathrm{o}}= & \mu p_{1}+O\left(\mu^{2}\right) \\
= & \mu \sqrt{x}\left(\frac{2}{3} x+1\right)+\frac{\mu}{2} \ln (1-\sqrt{x}) \\
& -\frac{\mu}{2} \ln (1+\sqrt{x})+\mu C_{1}+O\left(\mu^{2}\right)
\end{aligned}
$$

where $C_{1}$, the constant of integration, will be determined after matching. $C_{1}$ is multiplied by $\mu$, since the constant of integration should also be of $O(\mu)$.

\subsection{Inner expansion (Sharp-change region)}

Near to the moon $\left(M_{2}\right)$, the inner expansion is obtained by repeated application of the inner limit, which is defined as the limit when $\mu \rightarrow 0$ with the new position variable $\varphi=$ $\frac{(1-x)}{\mu}$ and the other dimensionless variables held fixed. To describe the inner solution near $x=1$, we write Eq. (35) in terms of the inner variable.

$$
\frac{\mathrm{d} p}{\mathrm{~d} \varphi}=\mu \sqrt{1-\mu \varphi}\left[1-\sqrt{\frac{\varphi}{\varphi+2(1-\mu \varphi)-1}}\right]
$$

Again, since the right-hand side is of $O(\mu)$, we seek an inner expansion of the perturbation in the form 


$$
p^{\mathrm{i}}=\sum_{n=1}^{\infty} \mu^{n} \pi_{n}
$$

Therefore upon substituting the inner expansion for $p$ in Eq. (39) and integrating under the inner limit process $\mu \rightarrow 0$ with $x$ fixed, the inner solution for the first term of the inner expansion is

$$
\begin{aligned}
\pi_{1}= & \varphi+(1-2 \mu)^{-3 / 2} \ln (\sqrt{(1-2 \mu) \varphi}+\sqrt{(1-2 \mu) \varphi+1}) \\
& -(1-2 \mu)^{-1} \sqrt{\varphi(\varphi+1)-2 \mu \varphi^{2}}+C_{2}
\end{aligned}
$$

where $C_{2}$ is a constant to be determined from matching.

\subsection{Matching process}

The general principles of matching are discussed in Refs. 7-10. To perform the second-order matching, we use a generalized form of the matching principle.

\section{The inner expansion of (the outer expansion) equals}

$$
\text { the outer expansion of (the inner expansion). }
$$

Thus we let

$\left(p^{\mathrm{o}}\right)^{\mathrm{i}}=$ the inner expansion of (the outer expansion) and

$$
\left(p^{\mathrm{i}}\right)^{\mathrm{o}}=\text { the outer expansion of (the inner expansion) }
$$

After the replacement of the inner and outer variables for the outer and inner expansions, respectively, a direct application of series expansions results in

$$
\begin{gathered}
\left(p^{\mathrm{o}}\right)^{\mathrm{i}}=\mu \sqrt{1-\mu \varphi}\left(\frac{2}{3}(1-\mu \varphi)+1\right)+\frac{\mu}{2} \ln (1-\sqrt{1-\mu \varphi})-\frac{\mu}{2} \ln (1+\sqrt{1-\mu \varphi})+\mu C_{1}+O\left(\mu^{2}\right) \\
=\frac{2 \mu}{3}\left(1-\frac{3}{2} \mu \varphi+\cdots\right)+\mu\left(1-\frac{1}{2} \mu \varphi+\cdots\right)+\frac{\mu}{2} \ln \mu+\frac{\mu}{2} \ln \varphi-\mu \ln 2+\mu C_{1}+O\left(\mu^{2}\right) \\
\left(p^{\mathrm{i}}\right)^{\mathrm{o}}=\mu\left[\frac{1-x}{\mu}+(1-2 \mu)^{-3 / 2} \ln \left(\sqrt{(1-2 \mu) \frac{1-x}{\mu}}+\sqrt{\left.(1-2 \mu) \frac{1-x}{\mu}+1\right)}\right.\right. \\
\left.-(1-2 \mu)^{-1} \sqrt{\frac{1-x}{\mu}\left(\frac{1-x}{\mu}+1\right)-2 \frac{(1-x)^{2}}{\mu}}+C_{2}\right]+O\left(\mu^{2}\right) \\
=-\mu(1-x)-\frac{\mu}{2}+\mu \ln 2+\frac{\mu}{2} \ln \frac{1-x}{\mu}+\mu C_{2}+O\left(\mu^{2}\right)
\end{gathered}
$$

By equating Eqs. (44) and (45) according to the matching principle and taking the limit, $\mu \rightarrow 0$, we have the relation for the constants of integration $C_{1}$ and $C_{2}$.

$$
C_{1}=2 \ln 2-\frac{1}{2} \ln \mu-\frac{13}{6}+C_{2}
$$

The common part $p_{00}$ is

$$
p_{00}=\mu\left[\frac{5}{3}-(1-x)-\ln 2+\frac{1}{2} \ln \mu+\frac{1}{2} \ln \varphi+C_{1}\right]+O\left(\mu^{2}\right)
$$

After using the generalized form of the matching principle, a composite solution uniformly valid over the whole trajectory for the perturbation $p$ subject to the initial condition (36) can be obtained as

$$
\begin{aligned}
p_{\mathrm{c}}= & p^{\mathrm{o}}+p^{\mathrm{i}}-p_{00} \\
= & \mu\left[\sqrt{x}\left(\frac{2 x}{3}+1\right)-\ln (1+\sqrt{x})+\varphi\right. \\
& \left.-(1-2 \mu)^{-1} \sqrt{\varphi(\varphi+1)-2 \mu \varphi^{2}}\right] \\
& +\mu(1-2 \mu)^{-3 / 2} \ln (\sqrt{(1-2 \mu) \varphi} \\
& +\sqrt{(1-2 \mu) \varphi+1})+\mu(1-x)
\end{aligned}
$$

$$
-\frac{5 \mu}{3}+\mu \ln 2+\mu C_{2}+O\left(\mu^{2}\right)
$$

Thus the improved second-order composite solution denoted with superscript $c$ can be formed according to Eq. (34).

$$
t^{\mathrm{c}}=\frac{2}{3 \sqrt{2}} x^{3 / 2}+p_{\mathrm{c}}
$$

Therefore we obtain the second-order composite solution with constant terms absorbed by $C_{2}$ in Eq. (43).

$$
\begin{aligned}
t^{\mathrm{c}}= & \frac{2}{3 \sqrt{2}} x^{3 / 2}+\frac{\mu}{\sqrt{2}}\left[\sqrt{x}\left(\frac{2 x}{3}+1\right)-\ln (1+\sqrt{x})\right. \\
& \left.+\varphi-(1-2 \mu)^{-1} \sqrt{\varphi(\varphi+1)-2 \mu \varphi^{2}}\right] \\
& +\mu(1-2 \mu)^{-3 / 2} \ln (\sqrt{(1-2 \mu) \varphi}+\sqrt{(1-2 \mu) \varphi+1}) \\
& -\mu x+\mu C_{2}+O\left(\mu^{2}\right)
\end{aligned}
$$

Using the classical method of MAE, Nayfeh obtained the following solutions ${ }^{10)}$ denoted with subscript $\mathrm{N}$.

$t_{\mathrm{N}}^{\mathrm{o}}=\frac{2}{3 \sqrt{2}} x^{3 / 2}+\frac{\mu}{\sqrt{2}}\left[\frac{2}{3} x^{3 / 2}+\sqrt{x}-\frac{1}{2} \ln \frac{1+\sqrt{x}}{1-\sqrt{x}}\right]+O\left(\mu^{2}\right)$ 


$$
\begin{aligned}
t_{\mathrm{N}}^{\mathrm{i}}= & \frac{2}{3 \sqrt{2}}+\frac{\mu}{\sqrt{2}}\left[\frac{13}{6}-2 \ln 2+\frac{1}{2} \ln \mu\right. \\
& \left.-\sqrt{\varphi(\varphi+1)}+\sinh ^{-1} \sqrt{\varphi}\right]+O\left(\mu^{2}\right) \\
t_{\mathrm{N}}^{\mathrm{c}}= & \frac{2}{3 \sqrt{2}} x^{3 / 2}+\frac{\mu}{\sqrt{2}}\left[\frac{1}{2}-\ln 2+\frac{1}{2} \ln \mu\right. \\
+ & \left.\frac{2}{3} x^{3 / 2}+\sqrt{x}-\frac{1}{2} \ln (1+\sqrt{x})\right] \\
+ & \frac{\mu}{\sqrt{2}}\left[\varphi-\sqrt{\varphi(\varphi+1)}+\sinh ^{-1} \sqrt{\varphi}\right]+O\left(\mu^{2}\right)
\end{aligned}
$$

The improved second-order composite solution (50) will be investigated for various mass ratios for comparison with Nayfeh's solution (53) and the numerical solution.

\section{Numerical Examples}

The earth-to-moon trajectory ( $\mu=0.01214)$ is considered as the first example. Figure 2 presents a comparison of the variations of the dimensionless time as functions of the position for the earth-to-moon trajectory. It is seen that five curves almost overlap with each other for most parts of the trajectory except near the moon $(x=1)$. One may deduce that they are of insignificant difference. In fact,

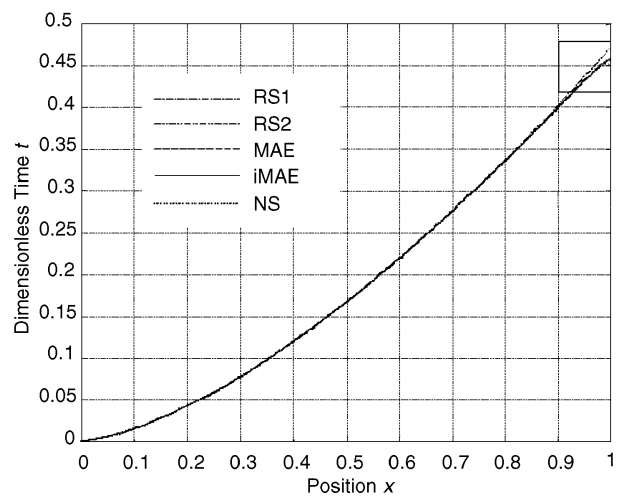

Fig. 2. The variations of the dimensionless time as functions of the position for the earth-to-moon trajectory with $\mu=0.01214$.

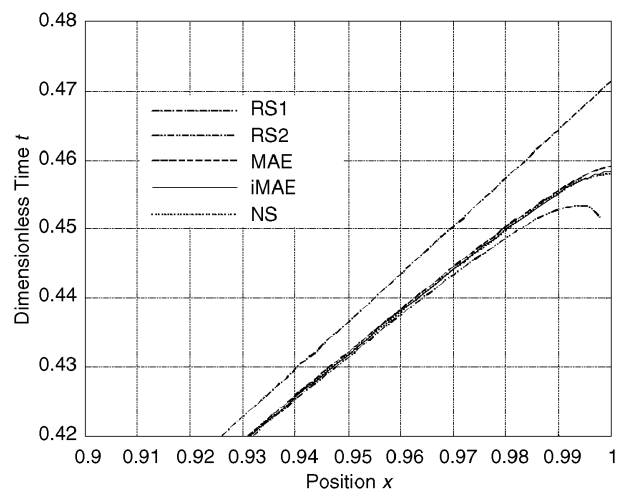

Fig. 3. Magnification of the box in Fig. 2. they have different errors. The box in the upper-right corner of the figure is magnified to investigate the detail in Fig. 3. The dash-dotted line (RS1) and dashed double-dotted (RS2) line denote the first- and second-order solutions using straightforward expansions (regular perturbation solutions), and the dashed line (MAE), solid line (iMAE), and dotted line (NS) represent solutions using the classical method of $\mathrm{MAE}$, the improved MAE, and pure numerical integration, respectively. We observe that when straightforward expansions are used, the second-order solution (RS2) is more accurate than the first-order solution (RS1) for the trajectory near the earth, and it becomes singular near the moon. The second-order solution using regular perturbation is not uniformly valid because it has a logarithmic singularity at the position of the moon, and higher-order solutions are increasingly more singular in that region. The curves of MAE, iMAE, and NS in Fig. 3 are almost identical, but we can still distinguish the differences between them. The errors of the classical MAE and improved MAE solutions with respect to the numerical solution are plotted in Fig. 4. The final errors of the dimensionless time are approximately $0.5 \times 10^{-4}$ and $5.0 \times 10^{-4}$ for iMAE and MAE solutions, respectively. It is almost 18.75 to 187.5 seconds of errors if translated into the regular time unit. We next considered the restricted threebody systems with a small value of the mass ratio $\mu$, but not as small as the earth-moon system, $\mu=0.075$ and a moderate value of $\mu=0.2$. The trajectories for the variations of the dimensionless time as functions of the position are plotted in Figs. 5 and 6 . Their errors with respect to the numerical solutions are plotted in Figs. 7 and 8. It is seen that the iMAE solutions almost coincide with numerical solutions for most parts of the trajectories, especially for a small value of the mass ratio. The asymptotic solution obtained by use of the improved method gives a better accuracy than those using the classical method for wide ranges of the small parameter. To reveal the formation of the composite solution and the action of matching, Fig. 9 presents the outer, inner, and composite solutions for the perturbation variable $p$ with the mass ratio $\mu=0.075$. In this figure, the dash-dotted line and dashed double-dotted line denote

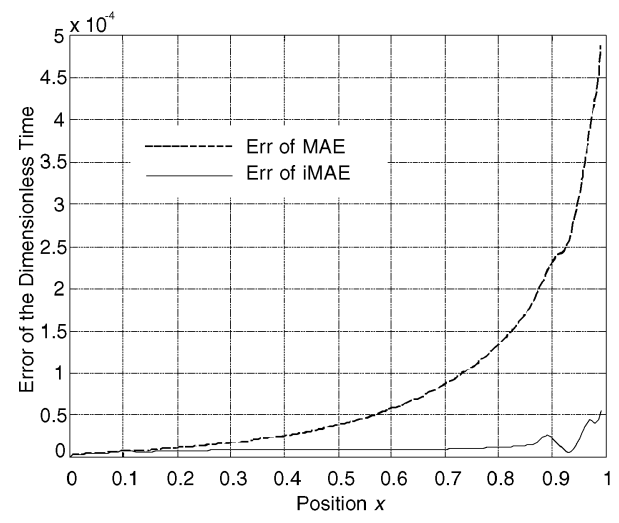

Fig. 4. The errors of the MAE and iMAE solutions with respect to the numerical solution for $\mu=0.01214$. 


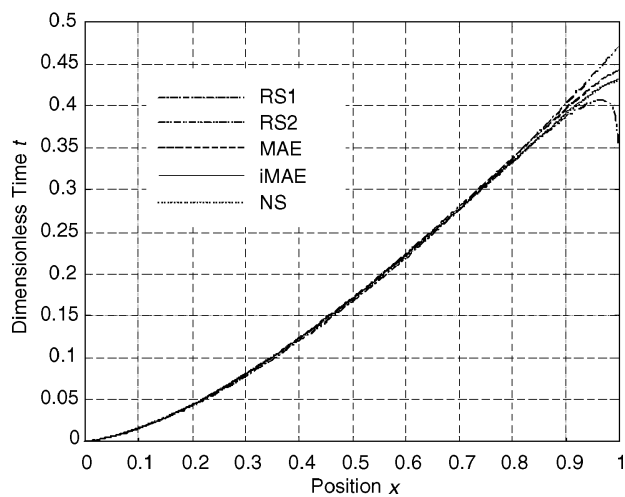

Fig. 5. The variations of the dimensionless time as functions of the position for $\mu=0.075$.

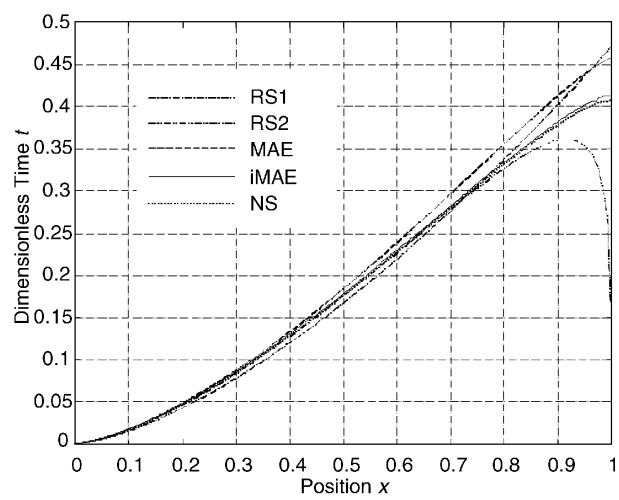

Fig. 6. The variations of the dimensionless time as functions of the position for $\mu=0.2$.

the outer and inner solutions, respectively, and the solid line is for the composite solution and the dotted line for the numerical solution. In particular, the dashed line denotes the common limit, Eq. (47), of the outer and inner expansions. Note that the common limit nearly coincides with the inner solution in the outer region (near the earth), but it approaches the outer solution in the inner region (near the moon). Thus according to the first equality of Eq. (48), the composite solution is formed by totaling the outer and inner expansions, then subtracting the common limit. It is seen that the composite solution $p_{\mathrm{c}}$ (solid line) shows excellent agreement in comparison with the numerical solution.

\section{Conclusions}

In this paper we apply the improved method of the matched asymptotic expansions technique for the limiting case of the restricted three-body problem. Analytical asymptotic solutions are obtained by the use of two alternative methods, namely, the classical MAE and the improved method of MAE. The asymptotic solutions are uniformly valid through the outer and inner regions. The major difference in these two methods is that a straightforward matching of the outer and inner expansions to higher-order terms is used in the classical MAE. On the other hand, in the improved technique, as presented in the paper, the second-

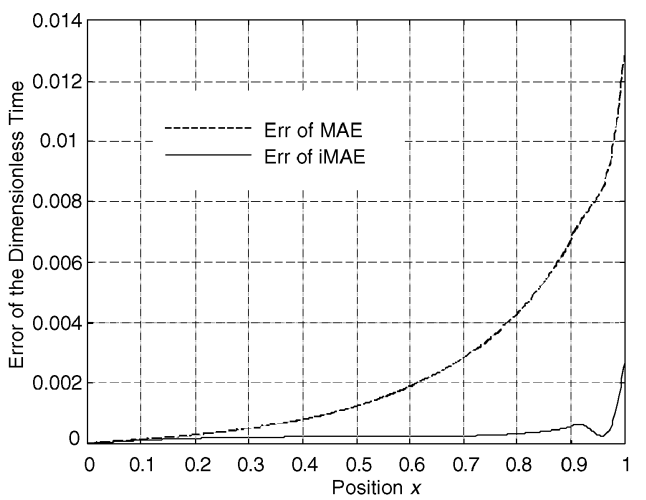

Fig. 7. The errors of the MAE and iMAE solutions with respect to the numerical solution for $\mu=0.075$.

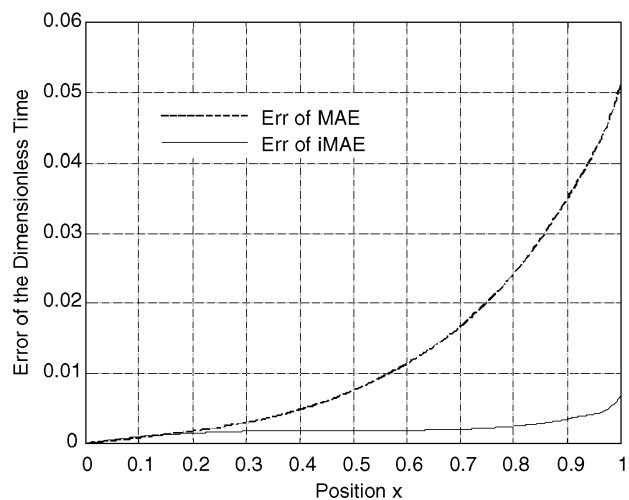

Fig. 8. The errors of the MAE and iMAE solutions with respect to the numerical solution for $\mu=0.2$.

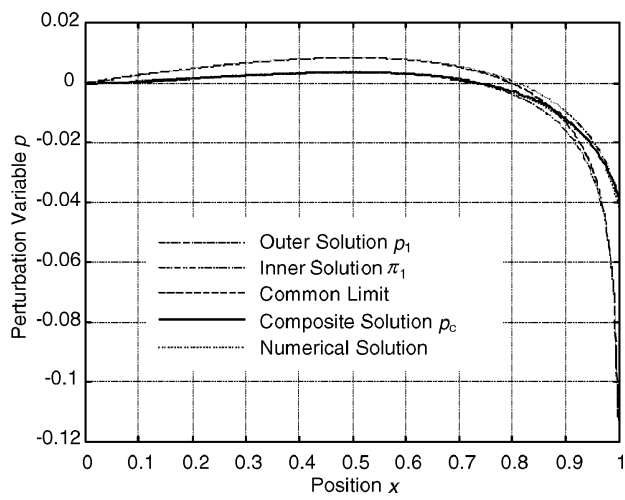

Fig. 9. The variations of the perturbation variable $p$ as functions of the position for $\mu=0.075$.

order solutions are derived by first generating perturbations between the first-order composite solutions and the exact solutions. Then the perturbation equations are integrated in the outer and inner regions for a second-order matching. Compared to the solutions obtained by the classical MAE and pure numerical integration over wide ranges of the small parameter, the second-order solutions obtained by this improved technique are very accurate. The improved MAE gives closer approximations to the exact solutions than the classical MAE does, as seen in Figs. 2-8. The reason is that in the matching of the second-order expansions, there is no 
crossover interference between different order expansions. Moreover, the equations of perturbation always describe the propagation errors between the composite solution and the exact solution with no approximation in the outer and inner regions. We have seen that the improved asymptotic solution for the limiting case of the restricted three-body problem provides a significant improvement over wide ranges of the small parameter $\mu$. This clearly demonstrates the applicability and feasibility of the improved method of MAE. The improved technique presented in this paper should be useful and amenable for further research.

\section{Acknowledgments}

The author wishes to thank Professor Nguyen X. Vinh and Professor Arthur F. Messiter for their useful suggestions. Moreover, the author would like to thank the National Science Council of the Republic of China for financially supporting this research under Contract NSC 89-2612-E-014-009.

\section{References}

1) Marchal, C. L.: The Restricted Three-Body Problem Revisited, Acta Astronautica, 47 (2000), pp. 411-418.

2) Jorba, A. and Villanueva, J.: Numerical Computation of Normal Forms around Some Periodic Orbits of the Restricted Three-Body Problem, Physica D, 114 (1998), pp. 197-229.

3) Krizek, M.: Numerical Experience with the Three-Body Problem, $J$.
Comput. Appl. Math., 63 (1995), pp. 403-409.

4) Celletti, A. and Froeschle, C.: Numerical Investigation of the BreakDown Threshold for a Restricted Three-Body Problem, Planet. Space Sci., 46 (1998), pp. 1535-1542.

5) Miele, A. and Mancuso, S.: Optimal Trajectories for Earth-MoonEarth Flight, Acta Astronautica, 49 (2001), pp. 59-71.

6) Prandtl, L.: Motion of Fluids with Very Little Viscosity, NACA Translation, No. 452, 1928.

7) Van Dyke, M.: Perturbation Methods in Fluid Mechanics, Academic Press, Inc., New York, 1964, pp. 77-97.

8) Kaplun, S., Lagerstrom, P. A., Howard, L. N. and Liu, C. S.: Fluid Mechanics and Singular Perturbations, Academic Press, Inc., New York, 1967, Chap. 2.

9) Kevorkian, J. and Cole, J. D.: Perturbation Methods in Applied Mathematics, Springer-Verlag, New York, 1981, pp. 179-185.

10) Nayfeh, A. H.: Perturbation Methods, John Wiley \& Sons, New York, 1973, pp. 110-139.

11) Lagerstrom, P. A.: Matched Asymptotic Expansions, Springer-Verlag, New York, 1988.

12) Lagerstrom, P. A. and Kevorkian, J.: Earth-to-Moon Trajectories in the Restricted Three-Body Problem, J. Mecanique, 2 (1963), pp. 189218.

13) Breakwell, J. V. and Perko, L. M.: Matched Asymptotic Expansions, Patched Conics, and the Computation of Interplanetary Trajectories, Progress in Astronautics and Aeronautics, Vol. 17, ed. by Duncombe, R. L. and Szebehely, V. G., Academic Press, New York, 1966, pp. 159-182.

14) Kuo, Z.-S. and Vinh, N. X.: Improved Matched Asymptotic Solutions for Three-Dimensional Atmospheric Skip Trajectories, J. Spacecraft Rockets, 34 (1997), pp. 496-502.

15) Kuo, Z.-S. and Yang, Y.-L.: Hypervelocity Trajectories of Ballistic Entry Using Improved Matched Asymptotic Expansions, Trans. Jpn. Soc. Aeronaut. Space Sci., 42 (2000), pp. 17-24. 